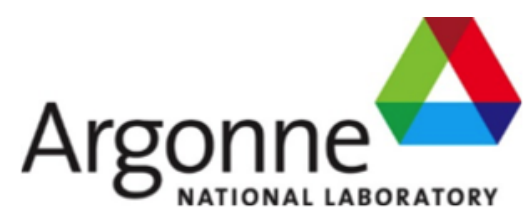

\title{
Comprehensive Margin Assessment of the ASME Section III, Division 5, Class A Primary Load Design Rules
}

Applied Materials Division 
About Argonne National Laboratory

Argonne is a U.S. Department of Energy laboratory managed by UChicago Argonne, LLC under contract DE-AC02-06CH11357. The Laboratory's main facility is outside Chicago, at 9700 South Cass Avenue, Argonne, Illinois 60439. For information about Argonne and its pioneering science and technology programs, see www.anl.gov.

\section{DOCUMENT AVAILABILITY}

Online Access: U.S. Department of Energy (DOE) reports produced after 1991 and a growing number of pre-1991 documents are available free at OSTI.GOV (http://www.osti.gov/), a service of the U.S. Dept. of Energy's Office of Scientific and Technical Information

Reports not in digital format may be purchased by the public from the

National Technical Information Service (NTIS):

U.S. Department of Commerce

National Technical Information Service

5301 Shawnee Rd

Alexandria, VA 22312

www.ntis.gov

Phone: (800) 553-NTIS (6847) or (703) 605-6000

Fax: (703) 605-6900

Email: orders@ntis.gov

Reports not in digital format are available to DOE and DOE contractors from the Office of Scientific and Technical Information (OSTI)

U.S. Department of Energy

Office of Scientific and Technical Information

P.O. Box 62

Oak Ridge, TN 37831-0062

www.osti.gov

Phone: (865) 576-8401

Fax: (865) 576-5728

Email: reports@osti.gov

\section{Disclaimer}

This report was prepared as an account of work sponsored by an agency of the United States Government. Neither the United States Government nor any agency thereof, nor UChicago Argonne, LLC, nor any of their employees or officers, makes any warranty, express or implied, or assumes any legal liability or responsibility for the accuracy, completeness, or usefulness of any information, apparatus, product, or process disclosed, or represents that its use would not infringe privately owned rights. Reference herein to any specific commercial product, process, or service by trade name, trademark, manufacturer, or otherwise, does not necessarily constitute or imply its endorsement, recommendation, or favoring by the United States Government or any agency thereof. The views and opinions of document authors expressed herein do not necessarily state or reflect those of the United States Government or any agency thereof, Argonne National Laboratory, or UChicago Argonne, LLC. 


\section{Comprehensive Margin Assessment of the ASME Section III, Division 5, Class A Primary Load Design Rules}

Applied Materials Division

Argonne National Laboratory

September 2021

Prepared by

A. Nicolas, Argonne National Laboratory M. C. Messner, Argonne National Laboratory

T.-L. Sham, Idaho National Laboratory 



\section{Abstract}

This report provides a comprehensive margin assessment of the ASME Section III, Division 5, Subsection HB, Subpart B rules for the design of high temperature reactor components against load controlled stress limits. These rules, often called the Code primary load design rules, provide protection against creep rupture and plastic collapse under steady conditions. The method adopted here is to compare the deterministic ASME design life, according to the primary load design rules, against the expected, statistical service life of the component, considering creep rupture as the relevant failure mode. The report provides the design margin of the ASME rules in terms of the probability of premature failure - the probability that the component fails in service before reaching the ASME design life. To complete this assessment, the report describes the development of a statistical creep life assessment procedure accounting for variations in the component loading, material creep rate, and the material rupture time. This includes a novel method for correlating creep rupture data using a Gaussian process accounting for heat-to-heat variation in the Larson-Miller timetemperature parameter. The report applies the complete margin assessment process to quantify the design margin inherent in the ASME primary load design rules as a function of temperature for relevant component geometries and materials. The overall conclusion is that the ASME rules are conservative, providing designs with very small probability of premature failure, but some optimization of the Code design rules is possible to provide a more uniform design margin. 

Comprehensive Margin Assessment of the ASME Section III, Division 5, Class A Primary Load Design Rules

September 2021

\section{Table of Contents}

Abstract $i$

Table of Contents $\quad$ iii

List of Figures $\quad$ V

List of Tables $\quad$ vii

1 Introduction 1

2 Probabilistic Margin Assessment 5

2.1 Overview . . . . . . . . . . . . . . . . . . 5

2.2 Rupture Life Distribution . . . . . . . . . . . . . . . . . . . 5

2.3 Pressure Vessel Design . . . . . . . . . . . . . . . . . . . . . . . 7

2.4 Pressure Vessel Stress Calculations . . . . . . . . . . . . . . . . . . . . 7

2.5 Power-Law creep Parameters . . . . . . . . . . . . . . . . . . . . . 10

2.6 Stress Variability and Rupture Time Probability . . . . . . . . . . . . . . . . 11

2.7 Margin Assessment for ASME design rules . . . . . . . . . . . . . . . . . . 12

2.8 Discussion . . . . . . . . . . . . . . . . . . . . . . . . . . 13

3 Improved Gaussian Process Modeling of Creep Rupture Data 17

3.1 Overview . . . . . . . . . . . . . . . . . . . 17

3.2 Gaussian Process Regression . . . . . . . . . . . . . . . . . . . . . . . 17

3.3 Gaussian Process Model for Creep Rupture . . . . . . . . . . . . . . . . . . . 19

3.4 Examples . . . . . . . . . . . . . . . . . . . . . . . . . . 19

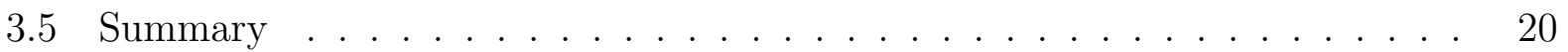

4 Conclusions 23

4.1 Meaningful Probabilities . . . . . . . . . . . . . . . . . 23

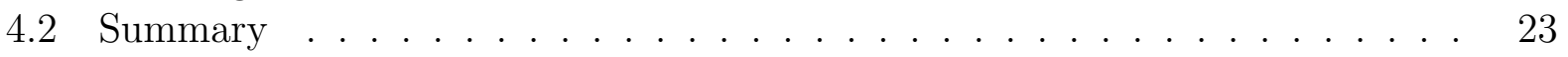

$\begin{array}{ll}\text { Bibliography } & 29\end{array}$ 



\section{List of Figures}

1.1 Illustration of the probability of premature failure $(F)$ on a probability distribution function (a) and a cumulative distribution function (b) describing component failure as a function of time. . . . . . . . . . . . . .

2.1 Code needed to run Gaussian Process fitting using the GPy module in Python. The script remains the same for all materials. . . . . . . . . . . . . . 6

2.2 Command in GPy needed to extract the distribution of the LMP at any given stress. . . . . . . . . . . . . . . . . .

2.3 Cumulative Distribution Functions of the LMPs through the entire stress range on (a) 316 Stainless Steel and (b) Grade 91 Steel. The blue scatterplots show the full experimental data, and the red plotted lines show the GP fit mean and prediction bands. . . . . . . . . . . . . . . 7

2.4 Simple pressure vessel geometry (cross section) and boundary conditions. . . 8

2.5 Comparison of the von Mises stresses from the Stokes model and the structural model for a stainless steel 316 vessel. . . . . . . . . . . . . . . . . . . . 10

2.6 Experimental and fitted correlations of the power-law creep distribution parameters (mean $\mu$ and standard deviation $\sigma$ ) for both 316 Stainless Steel and Grade 91 Steel . . . . . . . . . . . . . . . . . . . . .

2.7 Histogram with fitted Gaussian distribution of the maximum stresses for all Monte Carlo iterations in a SS316H vessel designed at $650{ }^{\circ} \mathrm{C} \ldots$. . . . . . . 11

2.8 (a) PDF and (b) CDF of the time to rupture for stainless steel 316 vessel at $650{ }^{\circ} \mathrm{C}$. The red line shows the design life of the vessel. . . . . . . . . . . . 12

2.9 Rupture data along with a yellow scatterplot that shows the mean stress and mean LMP at each evaluated temperature in Table 2.2 and Table 2.3. . . . . 15

2.10 Probabilities from Tables 2.2 and 2.3 plotted as a function of temperature. . 16

3.1 Example of Gaussian process regression. . . . . . . . . . . . . . . . . 18

3.2 Comparison between classical Larson-Miller polynomial regression and the Gaussian process regression model for $316 \mathrm{H}$. . . . . . . . . . . . . . . . . . . 21

3.3 Comparison between classical Larson-Miller polynomial regression and the Gaussian process regression model for Grade 91. . . . . . . . . . . . . . . . . 21

3.4 Comparison between classical Larson-Miller polynomial regression and the Gaussian process regression model for Alloy 617. . . . . . . . . . . . . . .

3.5 Comparison between classical Larson-Miller polynomial regression and the Gaussian process regression model for Alloy 740H. . . . . . . . . . . . . . . . 

Comprehensive Margin Assessment of the ASME Section III, Division 5, Class A Primary Load Design Rules

September 2021

\section{List of Tables}

2.1 Optimized values of RBF kernels used in GP fit . . . . . . . . . . . . . 6

2.2 Temperatures and resulting POFs for 316 Stainless Steel . . . . . . . . . . . 13

2.3 Temperatures and resulting POFs for Grade 91 Steel . . . . . . . . . . . . . 14

3.1 Summary of the 10-fold cross-validation test, comparing the GP and polynomial regression approaches. . . . . . . . . . . . . . . . 20

4.1 The size of the rupture database for the four materials considered in this report. 23 



\section{Introduction}

Section III, Division 5, Subsection HB, Subpart B provides design and construction rules for Class A high temperature nuclear reactor structural components. The Code rules encompass a variety of design conditions including:

1. Time independent failure caused by plastic collapse under steady load

2. Time dependent creep failure under steady load

3. Time independent and time dependent, cyclic excessive strain accumulation

4. Time dependent, cyclic creep-fatigue damage accumulation

5. Both time independent and time dependent buckling failure.

Of these design criteria, the Code combines the check against time-independent plastic collapse and time-dependent creep failure under steady loads into a set of primary load design rules. Historically, these primary load design rules are significant because they correspond to traditional non-nuclear design practice and were used for component sizing and preliminary design.

The primary load design rules are inherently an allowable stress criteria (ASC) approach - the designer analyzes the component and compares the stresses to allowable stresses set by the cognizant ASME Code Committees based on a careful evaluation of key material data. The difference between the high temperature design approach and other ASC design methods is that the stresses compared to the allowable stress values are the "load-controlled" stresses. At least for high temperature design, these load controlled stresses mimic the stationary creep stress distribution (at low temperatures they would mimic the fully-plastic stress distribution). Historically it was difficult to determine the stationary creep stress distribution directly and so the Code provides a load classification process for approximating the stationary stresses given the results of a linear elastic stress analysis.

The Class A primary load design criteria require evaluating the component against two allowable stresses: $S_{o}$ which does not dependent on time (though generally based on 100,000 hour extrapolated creep properties) to replicate non-nuclear design practice and the timedependent $S_{m t}$, based on time-dependent creep properties and, for short times, time independent strength. The time-dependent $S_{m t}$ rules embed a key concept of the ASME Section III, Division 5 design approach - the idea of a design life. This concept recognizes that degradation and failure are inevitable for high temperature operation and so Code components are designed to past a finite amount of time - the component design life.

Past studies and applications of the Code suggest the ASME Code rules produce safe, perhaps overly-conservative component designs (c.f. [1] and related volumes). However, the exact design margin inherent in the Code rules has not be quantified. This report describes a comprehensive margin assessment of the Section III, Division 5, Subsection HB, Subpart B Class A primary load design rules.

Given the importance of the design life concept and the fact that the $S_{m t}$ allowable stress tends to control designs over the $S_{o}$ allowable stress, this report focuses on the timedependent, $S_{m t}$ primary load design rules. The allowable stress $S_{m t}$ is defined as the lesser of the time independent allowable stress $S_{m}$, defined as the lesser of 
1. From $67 \%$ to $90 \%$ (depending on the material) of the ASME material yield strength

2. $33 \%$ of the ASME material tensile strength

and the time-dependent allowable stress $S_{t}$, defined as the lesser of

1. The tensile strength at temperature divided by a factor of 1.1

2. $100 \%$ of the average stress to cause $1 \%$ total strain over the design life

3. $80 \%$ of the minimum stress to cause the initiation of the tertiary creep over the design life

4. $67 \%$ of the minimum stress to cause creep rupture over the design life.

Code committee practice is to use an approximate $95 \%$ lower confidence bound on the data (i.e. 1.65 times the standard error) to establish a minimum property.

Most future reactor components will operate in the regime where the time dependent part of the allowable stress controls the design. Of the three criteria making up the allowable stress $S_{t}$, only creep rupture is a real failure mechanism. The other two criteria provide service limits and approximately guard against multiaxial failure. Therefore, the focus of this report is determining the margin of the ASME rules against creep rupture under the stationary creep stress distribution.

The concept of design margin is somewhat nebulous. The general idea of margin is the difference between when a component would actually fail under service conditions versus the limits provided by the design rules. For low temperature structural design, margin can often be expressed as a load factor - i.e. the component could survive loads in excess of the design loads by some quantifiable amount. However, given that creep failure is both inevitable and time-dependent this concept does not translate to high temperature design.

Instead, we focus on the probability of premature failure $F$ defined as the probability that the component fails before it reaches the ASME design life. This concept embeds both the design goal of the ASME Code rules - that the rules provide a reasonable assurance that the service life will be in excess of the design life - and the time-dependent, inevitable nature of failure under high temperature creep conditions.

Figure 1.1 illustrates the concept of the probability of premature failure. At a fixed temperature, the concept treats the failure of the structure as a probability distribution over time, with support $[0, \infty]$, expressed with a probability distribution function $p_{f}(t)$. The event of interest is the failure of the component by creep rupture which, the ASME Code approximates as the failure of the first material point (see discussion below). This probability distribution is proper in that failure will eventually occur, i.e.

$$
\int_{0}^{\infty} p_{f}(t) d t=1
$$

Given this probability distribution, the probability of premature failure is the cumulative probability up to the design life, i.e.

$$
F=\int_{0}^{t_{d e s i g n}} p_{f}(t) d t
$$




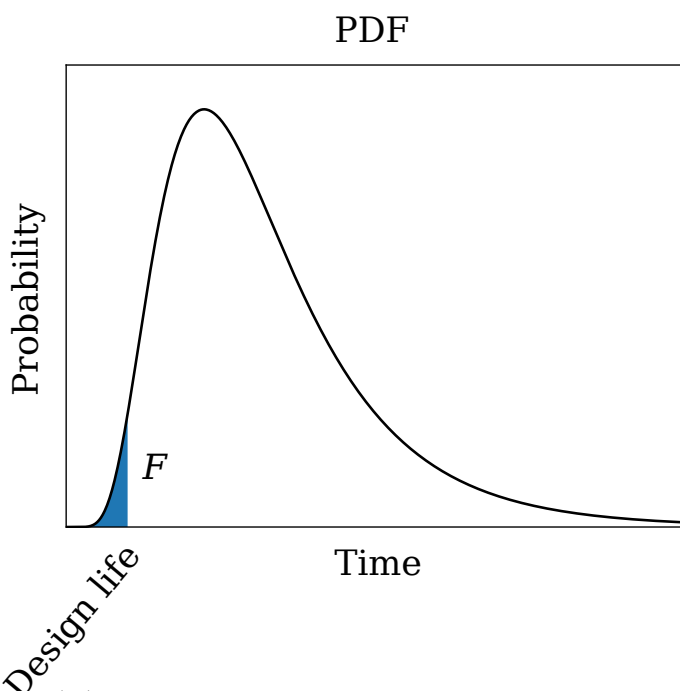

(a)

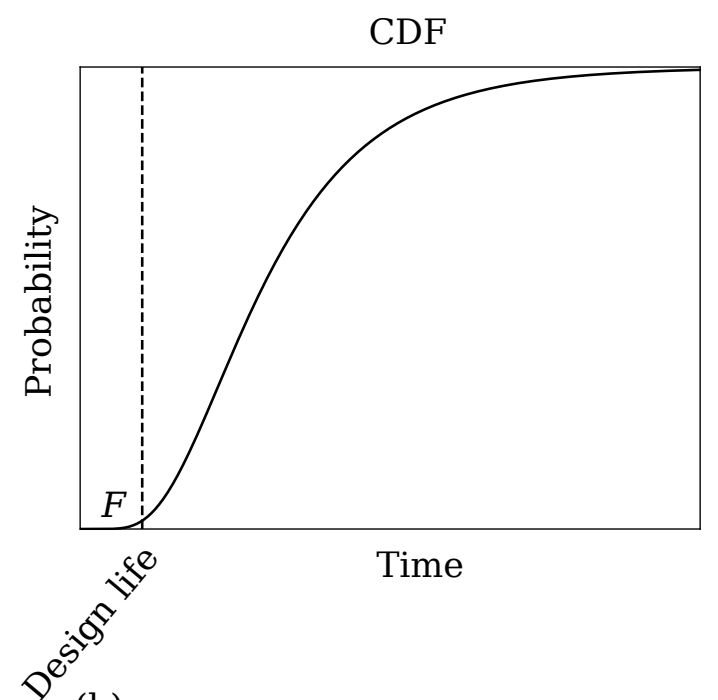

(b)

Figure 1.1: Illustration of the probability of premature failure $(F)$ on a probability distribution function (a) and a cumulative distribution function (b) describing component failure as a function of time.

This measure mathematically expresses the chance that failure occurs in service before the component reaches the target design life. Using $F$ as a measure of design margin means that smaller probabilities indicate a better, larger design margin. We could equally work with the component reliability defined as

$$
R=1-F
$$

in which case larger values indicate greater margin. However, this work uses $F$ consistently.

Assessing the margin of a particular component then requires two analyses: a deterministic ASME design analysis to determine the maximum allowable component design life $t_{\text {design }}$ and a statistical analysis based on creep rupture information to determine $p_{f}$. From this information the probability of premature failure follows from Eq. 1.2.

There are very few methods currently available for high temperature statistical design against creep [2-4]. As such, this report describes a novel method developed at Argonne National Laboratory to determine the probability distribution $p_{f}$ given the component geometry, statistics describing the expected component loads, material minimum creep rate data, and material stress rupture data. This design approach, described in Chapter 2, is comprehensive in that it can be applied to any component, including different combinations of material, design life, and temperature. We use this process to assess the margin inherent in the ASME rules over the complete temperature range for two key materials: $316 \mathrm{H}$ stainless steel and Grade 91 steel.

The probabilistic design approach mirrors the ASME primary load rules in that it uses the stationary creep stress distribution to evaluate the component against creep rupture under the steady temperature distribution. This process neglects the complications included in a full high temperature component structural evaluation which would include: 
1. The effects of varying load and temperature, both in damage accumulated during transient creep and for cyclic load, including creep-fatigue damage.

2. Similarly, damage accumulates through the component transient temperature history, not just at the steady state temperatures.

3. The residual service life left in the component after the initiation of creep damage. The analysis here stops when the analysis indicates one point in the structure would reach creep failure, as judged by indexing to creep rupture experimental data. However, many component geometries have redundant load paths and so this time would only correspond to the initiation of a creep crack. The time required to grow this creep crack to cause some service failure, like leaking, or the collapse of the component is residual component resistance not accounted for in either the ASME primary load design approach or in the statistical analysis.

Despite these limitations, the statistical assessment approach developed here is adequate to assess the ASME primary load design rules, as it mirrors the ASME deterministic design method, and is simple enough to be tractable even for complex component geometries. 
Comprehensive Margin Assessment of the ASME Section III, Division 5, Class A Primary Load Design

Rules

September 2021

\section{Probabilistic Margin Assessment}

\subsection{Overview}

This chapter presents a comprehensive probabilistic margin assessment of the ASME BPVC primary load design rules for Class A components in Section III Division 5. This chapter evaluates the design margin of several of the Class A materials for a simple, but representative, component geometry across the entire Division 5 elevated temperature range. The margin assessment applies a probabilistic life prediction methodology developed in previous work that accounts for the variability in material strength and deformation. A Gaussian process fit captures the the strength variability, and a Monte Carlo approach accounts for the variability of steady-state creep deformation parameters leading to variability in the stresses developed in a component. A very efficient method based on the analogy between viscous Stokes flow to steady-state creeping solid determines the steady-state stress distribution under primary load for the Monte Carlo approach. This chapter evaluates the design margin of several materials across a wide range of temperatures. The probabilistic margin assessment presented in this chapter gives an insight into the design margin in the currently deterministic ASME Section III, Division 5 primary load design rules for high temperature nuclear components.

\subsection{Rupture Life Distribution}

The life distribution model begins with the Larson Miller Parameter (LMP), calibrated to a large rupture database assembled for each of the materials evaluated. Equation (2.1) shows the LMP for 316 Stainless steel (SS316H), and Eqn. (2.2) shows the LMP for Grade 91 steel (modified 9Cr-1Mo):

$$
\begin{aligned}
& L M P=\frac{T}{1000}\left(\log _{10}(t)+20.54\right) \\
& L M P=\frac{T}{1000}\left(\log _{10}(t)+33.1\right)
\end{aligned}
$$

where $T$ is the temperature in kelvin $(\mathrm{K})$ and $t$ is the time in hours $(\mathrm{h})$. Dividing the LMP by a factor of 1000 provides data at a more numerically favorable scale. A least-square regression to a linear correlation between the LMP and log stress over the full rupture data set yields the LMP constants of 20.54 and 33.1, respectively. The values of the Larson Miller $C$ coefficient does change somewhat for polynomial correlations with a higher order, though not enough to significantly alter the rupture model predictions. However, Chapter 3 corrects this deficiency.

For each material, a Gaussian Process (GP) fit of the rupture data captures the uncertainty in rupture life at each stress state. The GP fit makes use of GPy, a framework available in Python as a module [5]. The GP fitting sets stresses as the independent variable, the LMPs as the dependent variable, and uses a 1-dimensional Radial Basis Function (RBF) kernel with an initial length scale of 10 and an initial variance of 4, based on the magnitude of the rupture data. The likelihood and inference method are Gaussian, with a likelihood variance of 0.05 and an exact inference method. Figure 2.1 shows the main script needed to 
Comprehensive Margin Assessment of the ASME Section III, Division 5, Class A Primary Load Design Rules

September 2021

perform a Gaussian process fit using the GPy module, for which $X$ is the stresses and $Y$ is the LMP values.

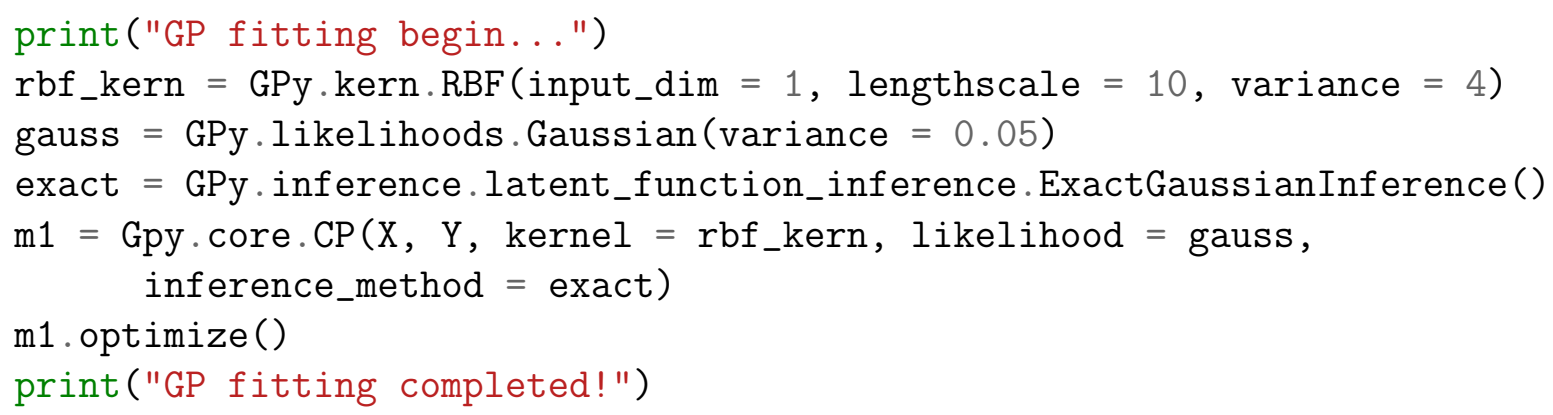

Figure 2.1: Code needed to run Gaussian Process fitting using the GPy module in Python. The script remains the same for all materials.

Table 2.1 shows the final variance and length scale values of the RBF kernel after GPy optimizes its values. The GP fit for each material used as training data any experimental data with a rupture life less than 10,000 hours, which resulted in fitting 1,344 points for 316 stainless steel and 1,561 for Grade 91 steel. The analysis used the GPy package because it can handle large datasets.

Table 2.1: Optimized values of RBF kernels used in GP fit

\begin{tabular}{rcc}
\hline Material & RBF variance & RBF length scale \\
\hline SS316H & 352.57 & 1.54 \\
Grade 91 & 1200.48 & 1.89 \\
\hline
\end{tabular}

Another advantage behind the GPy module is its ability to predict the distribution of the dependent variable given an unknown independent variable that was not originally among the experimental dataset [6]. The module therefore was capable of extracting the mean and the variance of the LMP at any queried stress using the command in Figure 2.2, therefore allowing to extract the marginal probability $P$ of the LMP at a specified rupture stress $P\left(L M P \mid \sigma_{i}\right)$, which is equivalent to the probability of the time to rupture at a specified rupture stress $P\left(t \mid \sigma_{i}\right)$ for a fixed temperature.

\section{lmp_gp, lmp_gpvar = m1.predict(s)}

Figure 2.2: Command in GPy needed to extract the distribution of the LMP at any given stress. 
Figure 2.3 shows, for each material, the GP fitted response, the $95 \%$ prediction intervals, and the cumulative distribution function (CDF) of all LMPs at each stress state. These responses plotted in red show that GP fitting captures the behavior of the material beyond the experimental data, albeit the uncertainty of such a behavior increases the further we query away from the data (dotted lines). In these images, The greyscale plot represents the $\mathrm{CDF}$ value that comes from the conditional probability $P\left(L M P \mid \sigma_{i}\right)$.
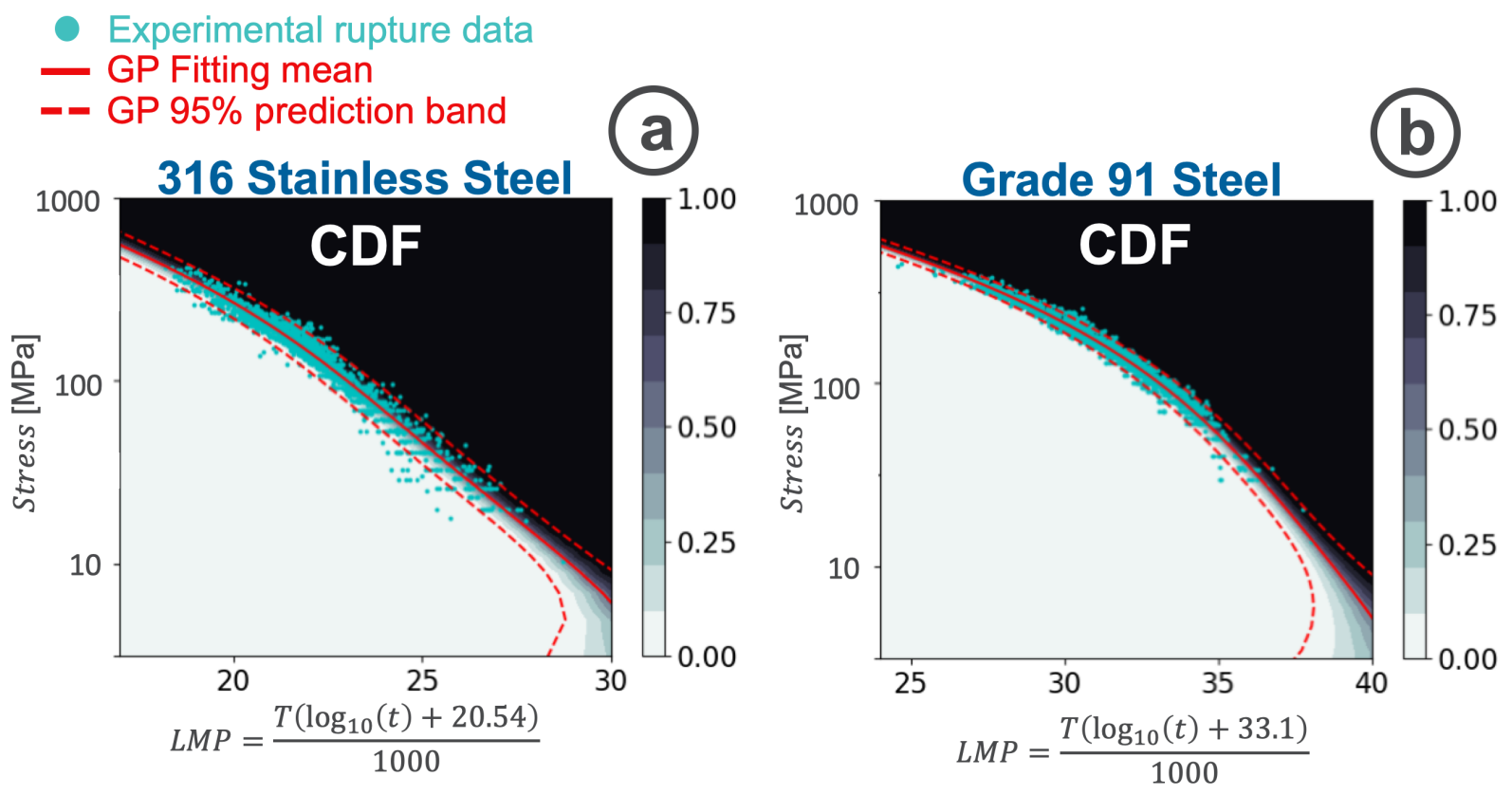

Figure 2.3: Cumulative Distribution Functions of the LMPs through the entire stress range on (a) 316 Stainless Steel and (b) Grade 91 Steel. The blue scatterplots show the full experimental data, and the red plotted lines show the GP fit mean and prediction bands.

\subsection{Pressure Vessel Design}

The primary load design rules in Section III Division 5, Subsection HB, Subpart B of the ASME Boiler Pressure and Vessel Code (BPVC) [7] allows to design a simple cylindrical pressure vessel for each material and temperature of interest, where each vessel has a design life of 100,000h and an inner pressure of $1 \mathrm{MPa}$. As a result, each temperature (and material) will result in a different minimum vessel thickness $t_{m}$ that is a function of the allowable stress $S_{m t}$. Figure 2.4 shows the axisymmetric cylindrical geometry of the pressure vessel, as well as the boundary conditions. The applied stress in the axial direction $\sigma_{z}$ is the analytical solution for a thin wall cylindrical vessel. This process results in a total of 15 different geometries for each material, one for each temperature evaluated at each material

\subsection{Pressure Vessel Stress Calculations}

To perform a large number of simulations in a reduced amount of time, this work models the steady state creep in the vessels as an incompressible Stokes flow with a stress-dependent 


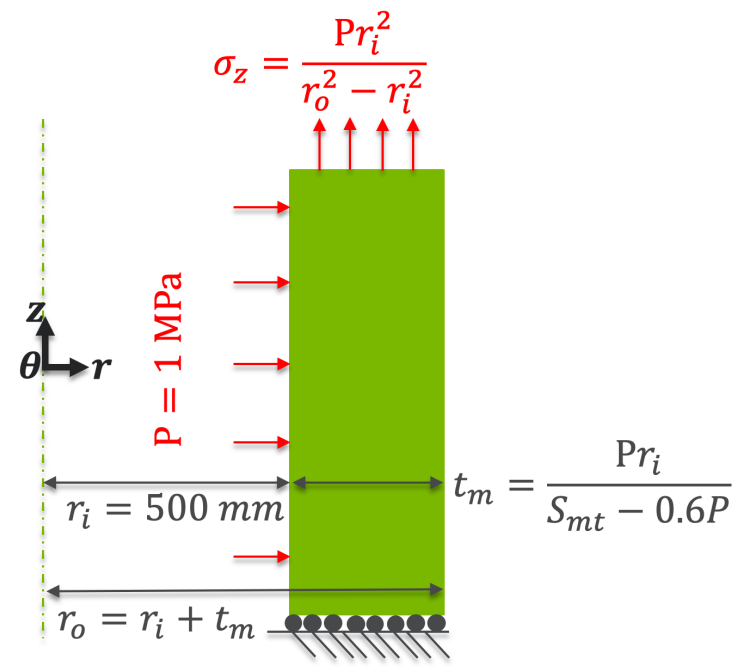

Figure 2.4: Simple pressure vessel geometry (cross section) and boundary conditions.

viscosity [8, 9]. The Stokes flow solution yields the steady stress distribution in the vessel in a matter of seconds, which is magnitudes faster when compared to the traditional solid mechanics transient analysis, for which run times may last from minutes to hours. In a steady-state Stokes flow, the power-law creep parameters determine the non-Newtonian viscosity in the fluid.

This work models the Stokes problem with the Navier-Stokes module [10] in MOOSE [11], using the Automatic Differentiation (AD) version of the module to calculate the Jacobians automatically. The model applies the Pressure-Stabilized Petrov Garlekin (PSPG) $[10,12]$ method to circumvent instabilities in the discretization of the Stokes equations. The simulations here apply a stabilization factor of $\alpha=0.01$. The creep model implements the traction form of the incompressible Navier-Stokes equations [10]:

$$
\begin{array}{r}
\rho\left(\frac{\partial \boldsymbol{u}}{\partial t}+\boldsymbol{u} \cdot \nabla \boldsymbol{u}\right)-\nabla \cdot \boldsymbol{\sigma}=\boldsymbol{f} \\
\nabla \cdot \boldsymbol{u}=0
\end{array}
$$

where $\boldsymbol{u}$ is the velocity, $\rho$ is the (constant) fluid density, $\boldsymbol{f}$ is the body force per unit volume, and $\boldsymbol{\sigma}$ is the total stress tensor. Assuming negligible inertial forces (i.e. the transient term and the convective term) results in the Stokes equations:

$$
\begin{aligned}
\nabla \cdot \boldsymbol{\sigma} & =\mathbf{0} \\
\nabla \cdot \boldsymbol{u} & =0
\end{aligned}
$$

where this equation also omits the body force.

The stress tensor $\boldsymbol{\sigma}$ is defined as:

$$
\boldsymbol{\sigma}=-P \boldsymbol{I}+\boldsymbol{\tau}
$$


where $P$ is the pressure, $\boldsymbol{I}$ is the identity tensor, and $\boldsymbol{\tau}$ is the viscous stress tensor of the material, defined as:

$$
\tau=G \dot{\gamma}
$$

where $\dot{\gamma}$ is the shear rate

$$
\dot{\gamma}=\frac{1}{2}\left(\nabla \boldsymbol{u}+\nabla \boldsymbol{u}^{T}\right)
$$

and $G$ is the viscosity of the material. A non-Newtonian viscosity describes power-law creep with:

$$
\mu=G_{0} \dot{\gamma}_{\text {eff }}^{m-1}
$$

where $G_{0}$ is the initial viscosity, $m$ is the thickening/thinning exponent (note that at $m=1$ the viscosity becomes Newtonian), and where $\dot{\gamma}_{\text {eff }}$ is the von Mises effective shear rate of the material [13], here in cylindrical coordinates:

$$
\dot{\gamma}_{\text {eff }}=\sqrt{\frac{2}{3}\left(2\left[\left(\frac{\partial u_{r}}{\partial r}\right)^{2}+\left(\frac{\partial u_{z}}{\partial z}\right)^{2}+\left(\frac{u_{r}}{r}\right)^{2}\right]+\left[\frac{\partial u_{r}}{\partial z}+\frac{\partial u_{z}}{\partial r}\right]^{2}\right)}
$$

Where the subscript indicates the component of the velocity field in the indicated direction. This work develops an AD material object within MOOSE to evaluate Equation 2.10 and Equation 2.11.

Substituting Equation 2.10 into Equation 2.8, and assuming that the shear rates are similar, the viscous stress is:

$$
\tau_{e f f}=G_{0} \dot{\gamma}_{e f f}^{m}
$$

with

$$
\tau_{e f f}=\sqrt{\frac{3}{2} \boldsymbol{\sigma}: \boldsymbol{\sigma}}
$$

Solving for the shear rate results in an equation analogous to the power-law definition:

$$
\dot{\gamma}_{\text {eff }}=A \tau_{\text {eff }}^{n}
$$

where:

$$
\begin{aligned}
n & =1 / m \\
A & =G_{0}^{-n}
\end{aligned}
$$

Therefore, the main input of the Stokes model becomes the power-law creep parameters $A$ and $n$, which in turn define the non-Newtonian viscosity parameters $\mu_{0}$ and $n$.

A transient structural simulation of a $316 \mathrm{H}$ steel cylindrical pressure vessel at $650^{\circ} \mathrm{C}$ verifies the applicability of the Stokes flow solution, in which the Stokes model takes takes $\sim 10$ seconds to run, as opposed to the transient simulation which takes $\sim 20$ minutes to run. On both models the power-law parameters are $A=7.32 \times 10^{-25}$ and $n=8.52$. The structural simulation spans 500,000 hours to approximate the steady state solution. The structural solution approaches the steady state creep stress distribution only asymptotically, but after 500,000 hours the stresses change only very slowly as a function of time. Figure 2.5a shows a comparison of the von Mises stresses that result from both models. Evaluating 
the von Mises stress described in Eq. (2.15) allows to relate the multiaxial behavior in the component to the uniaxial rupture model.

$$
\sigma_{v m}=\sqrt{\frac{\left(\sigma_{1}-\sigma_{2}\right)^{2}+\left(\sigma_{2}-\sigma_{3}\right)^{2}+\left(\sigma_{3}-\sigma_{1}\right)^{2}}{2}}
$$

where $\sigma_{1}, \sigma_{2}, \sigma_{3}$ are the three principal stresses.

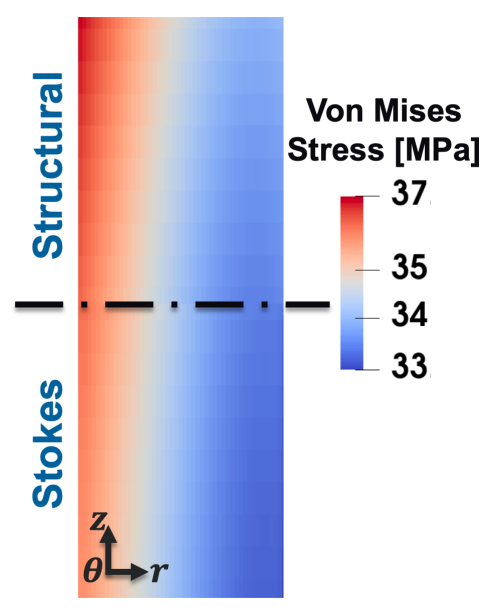

Figure 2.5: Comparison of the von Mises stresses from the Stokes model and the structural model for a stainless steel 316 vessel.

\subsection{Power-Law creep Parameters}

To obtain the steady-state stress distributions in the pressure vessels, the finite element (FE) model uses as input power-law creep parameters $A$ and $n$ from the steady-state creep rate dependence. Experimental Minimum Creep Rate (MCR) data of both 316 Stainless Steel and Grade 91 steel yield the mean $\mu$ and standard deviation $\sigma$ of the power-law creep parameters at each experimental temperature available. A linear correlation between the observed parameter values and the temperature allows to evaluate the mean and the standard deviation at temperatures not available in experimental data.

Figure 2.6 shows the linear correlations used to obtain the mean and standard deviation of the power-law creep parameters at any temperature.

One exception is the standard deviation $\sigma$ of the power-law creep parameters for $316 \mathrm{H}$, which does not have any significant trend for either $A$ nor $n(R<0.5)$. Therefore, the standard deviation at any temperature becomes the average value: 4.07 for $n$ and 9.42 for $\log _{10} A$.

For each temperature, the Gaussian distribution that results from the selected $\mu$ and $\sigma$ in Figure 2.6 generates random power law parameters, within 2 standard deviations from the mean, that become the input to the Monte Carlo analysis needed to capture the stress variability. 

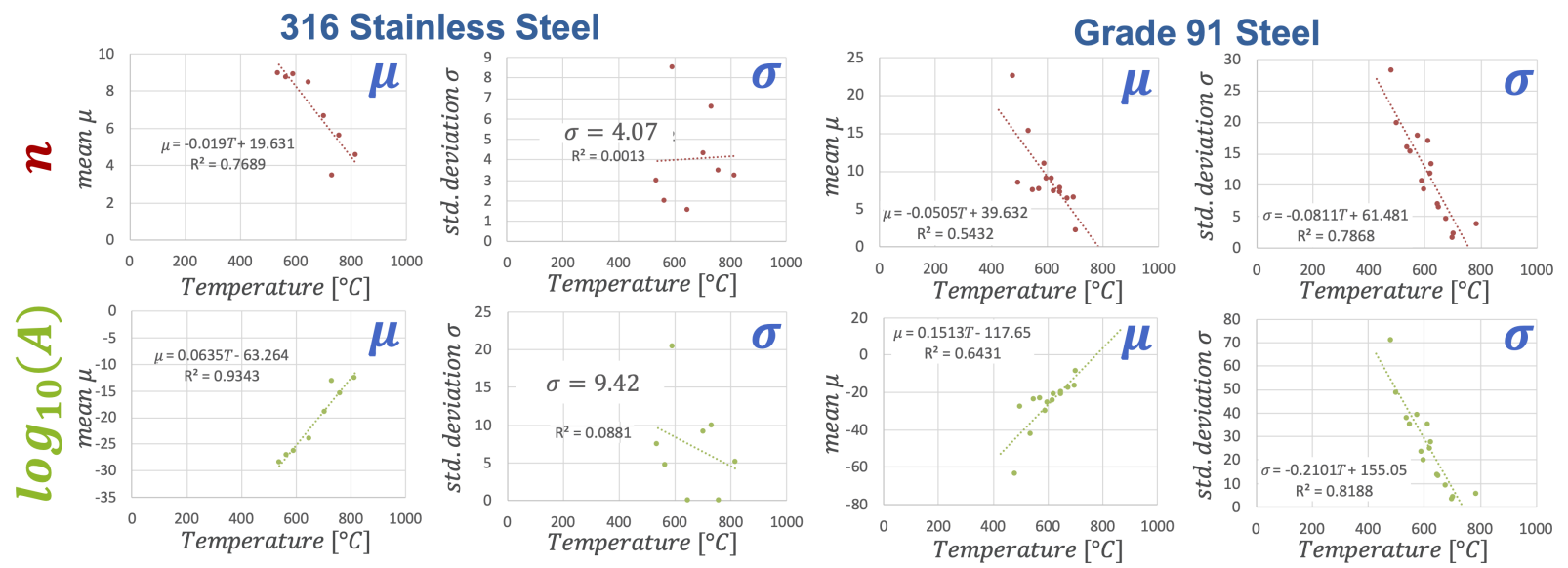

Figure 2.6: Experimental and fitted correlations of the power-law creep distribution parameters (mean $\mu$ and standard deviation $\sigma$ ) for both 316 Stainless Steel and Grade 91 Steel

\subsection{Stress Variability and Rupture Time Probability}

The Monte Carlo (MC) method allows to evaluate the maximum von Mises stress variability at each temperature. To apply this method for each temperature and each material, a random sampling of the power-law creep parameters from the distributions described in Section 2.5 generates between 500 1000 steady-state Stokes models with unique power-law creep parameter pairs per temperature and per material. Figure 2.7 shows an example of the stress variability obtained from $\mathrm{MC}$ for a $\mathrm{SS} 316 \mathrm{H}$ cylindrical pressure vessel designed to live for 100,000 hours at $650^{\circ} \mathrm{C}$. On all $\mathrm{MC}$ simulations, the maximum von Mises stress is always located at the inner wall of the vessel.

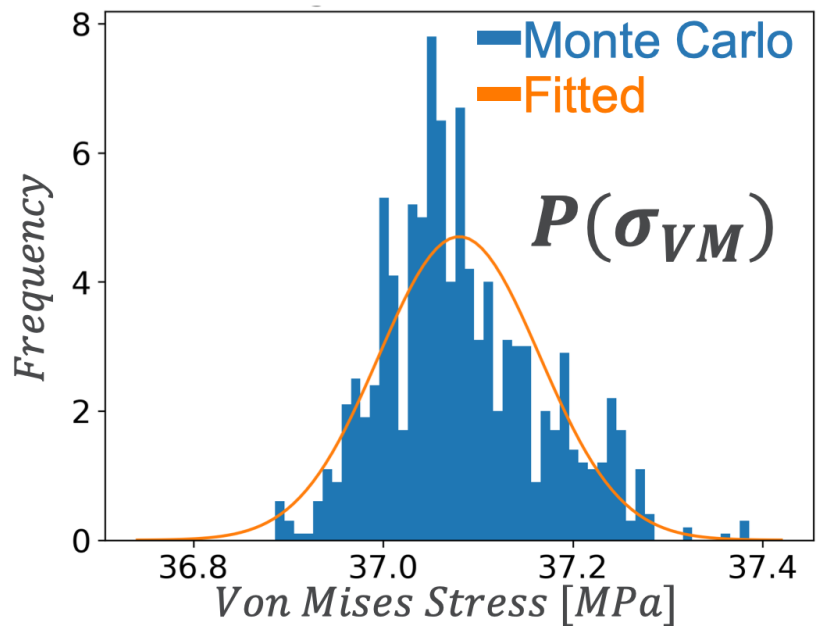

Figure 2.7: Histogram with fitted Gaussian distribution of the maximum stresses for all Monte Carlo iterations in a SS316H vessel designed at $650{ }^{\circ} \mathrm{C}$

The fitted Gaussian distribution describes the marginal probability of the maximum von Mises stress $P\left(\sigma_{v m}\right)$ in the vessel. The Gaussian distributions from MC converge at around 
Comprehensive Margin Assessment of the ASME Section III, Division 5, Class A Primary Load Design Rules

September 2021

250 samples for all materials and temperatures.

Obtaining the marginal probability allows the calculation of the rupture time probability in Eq. (2.16) [14]:

$$
p_{f}(t)=\sum_{i} P\left(t \mid\left(\sigma_{v m}\right)_{i}\right) P\left(\sigma_{v m}\right)
$$

where $P\left(t \mid\left(\sigma_{v m}\right)_{i}\right)$ is the probability of the time to rupture at a certain stress $\left(\sigma_{v m}\right)_{i}$. The GP fit in Section 2.2 calculates this probability at each stress. Integrating the the probability distribution function (PDF) that represents $P(t)$ will yield the CDF that quantifies the Probability of Failure (POF) at a certain time to rupture. Figure 2.8a shows the PDF and Fig 2.8b shows the $\mathrm{CDF}$ obtained for a $\mathrm{SS} 316 \mathrm{H}$ vessel at $650^{\circ} \mathrm{C}$ that resulted from the combination of the $P\left(\sigma_{v m}\right)$ in Fig.2.7 and the $P\left(t \mid\left(\sigma_{v m}\right)_{i}\right)$ in Fig.2.3. Figure 2.8 also shows the POF that is associated to the cylindrical pressure vessel design life of 100,000 hours $\left(10^{5} \mathrm{~h}\right)$. This POF value represents the probability that the pressure vessel fails before the ASME design life.
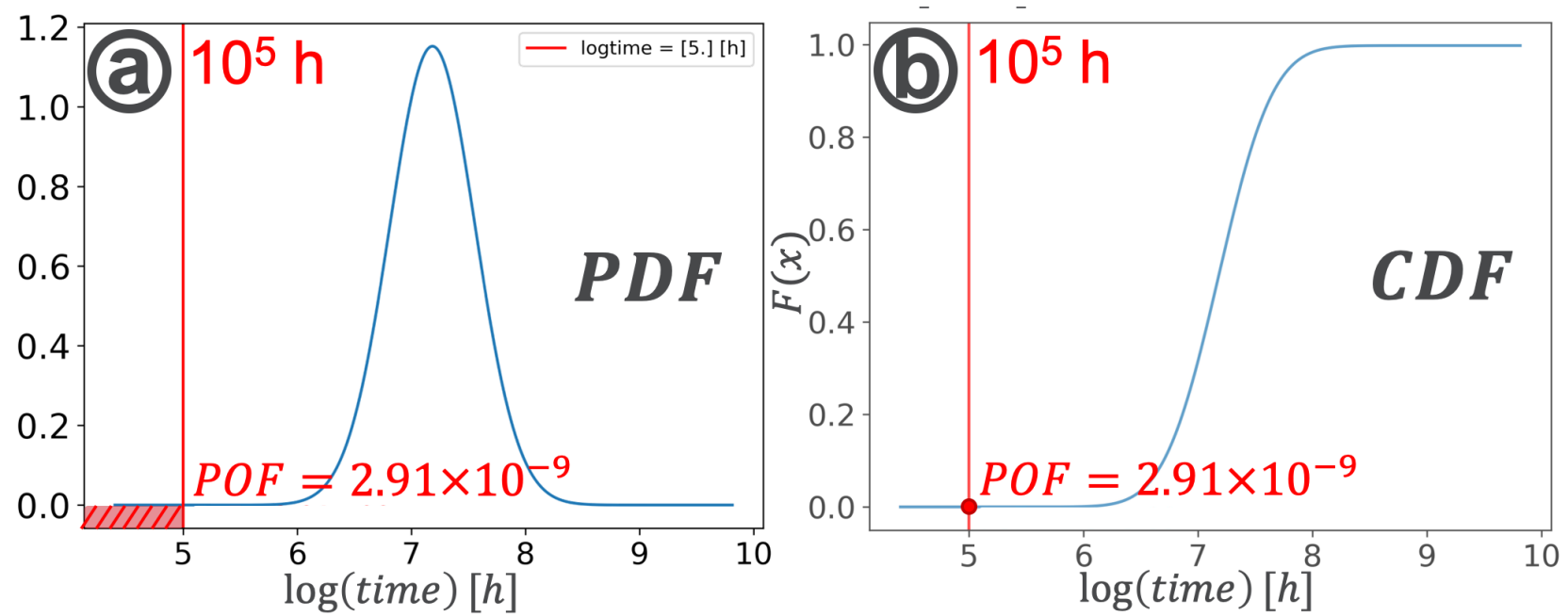

Figure 2.8: (a) PDF and (b) CDF of the time to rupture for stainless steel 316 vessel at 650 ${ }^{\circ} \mathrm{C}$. The red line shows the design life of the vessel.

\subsection{Margin Assessment for ASME design rules}

Following the procedure described previously for the entire ASME design temperature range resulted in the rupture time POFs in Table 2.2 and Table 2.3. The temperatures range every $20^{\circ} \mathrm{C}$ from $540^{\circ} \mathrm{C}$ to $800^{\circ} \mathrm{C}$ for 316 Stainless steel, and from from $380^{\circ} \mathrm{C}$ to $640^{\circ} \mathrm{C}$ for Grade 91 Steel. The ranges also include the maximum temperatures where experimental data is available for both materials: $816^{\circ} \mathrm{C}$ for 316 stainless steel and $650^{\circ} \mathrm{C}$ for Grade 91 steel.

The labels on the first columns relate to the labels in Fig.2.9 where they highlight, for each temperature, the equivalent LMP value and the average von Mises stress from the MC-based Gaussian distribution. Therefore, Fig.2.9 shows the distance between the final stresses in a cylindrical pressure vessel designed via ASME design rules (yellow scatterplot), 
Comprehensive Margin Assessment of the ASME Section III, Division 5, Class A Primary Load Design Rules

September 2021

Table 2.2: Temperatures and resulting POFs for 316 Stainless Steel

\begin{tabular}{ccc}
\hline Label & Temperature & POF \\
\hline (a) & 540 & $4.10 \mathrm{E}-09$ \\
(b) & 560 & $3.00 \mathrm{E}-07$ \\
(c) & 580 & $1.80 \mathrm{E}-08$ \\
(d) & 600 & $1.40 \mathrm{E}-10$ \\
(e) & 620 & $7.60 \mathrm{E}-11$ \\
(f) & 640 & $1.30 \mathrm{E}-10$ \\
(g) & 660 & $1.80 \mathrm{E}-10$ \\
(h) & 680 & $1.70 \mathrm{E}-10$ \\
(i) & 700 & $2.40 \mathrm{E}-11$ \\
(j) & 720 & $4.30 \mathrm{E}-11$ \\
(k) & 740 & $4.00 \mathrm{E}-12$ \\
(l) & 760 & $4.70 \mathrm{E}-10$ \\
(m) & 780 & $4.00 \mathrm{E}-07$ \\
(n) & 800 & $2.10 \mathrm{E}-04$ \\
(o) & 816 & $9.70 \mathrm{E}-03$ \\
\hline
\end{tabular}

the experimental rupture data (blue scatterplot), and the fitted rupture response (red solid line).

\subsection{Discussion}

This chapter performs a probabilistic margin assessment of the ASME Section III, Division 5 Primary Load Design Rules by applying an uncertainty-based life assessment on a series of cylindrical pressure vessels designed at different temperatures and materials. This assessment is based on experimental data, for both $316 \mathrm{H}$ and Grade 91 steel, that describes the uncertainty in both rupture and creep deformation. The rupture life probability calculation combines the stress distributions obtained via MC with the stress-dependent rupture time distribution obtained via GP fitting. The MC method relies on Stokes flow modeling to enable solving a large number of models in a short amount of time.

Figure 2.10 plots the probability of premature failure for the two materials as a function of temperature on a $\log$ scale to better display the data. This plot illustrates several trends:

1. The probabilities of premature failure are quite small for both materials. Though an adequate margin should be determined through broader consultation with the design community, regulators, and the public, the margin against rupture assessed here is quite significant. Recall from Chapter 1 that this probability is not a yearly frequency, i.e. a probability per year, but rather a cumulative probability over the design life.

2. Generally speaking, the design margins for the two materials considered here are similar. The ASME allowable stresses are consistently defined and so we should expect 
Table 2.3: Temperatures and resulting POFs for Grade 91 Steel

\begin{tabular}{ccc}
\hline Label & Temperature & POF \\
\hline (a) & 380 & $7.00 \mathrm{E}-29$ \\
(b) & 400 & $1.60 \mathrm{E}-21$ \\
(c) & 420 & $9.30 \mathrm{E}-15$ \\
(d) & 440 & $1.40 \mathrm{E}-09$ \\
(e) & 460 & $9.80 \mathrm{E}-07$ \\
(f) & 480 & $2.30 \mathrm{E}-04$ \\
(g) & 500 & $2.40 \mathrm{E}-07$ \\
(h) & 520 & $3.50 \mathrm{E}-06$ \\
(i) & 540 & $2.60 \mathrm{E}-07$ \\
(j) & 560 & $1.60 \mathrm{E}-08$ \\
(k) & 580 & $8.60 \mathrm{E}-09$ \\
(l) & 600 & $1.70 \mathrm{E}-08$ \\
(m) & 620 & $1.90 \mathrm{E}-10$ \\
(n) & 640 & $2.30 \mathrm{E}-11$ \\
(o) & 650 & $3.00 \mathrm{E}-11$ \\
\hline
\end{tabular}

there to be no significant differences in materials with comparably-sized experimental datasets. Both $316 \mathrm{H}$ and Grade 91 are well-established materials with large rupture databases and so we should expect similar design margins.

3. However, there are significant variations in the design margin as a function of temperature.

- At very low temperatures near the bottom of the creep range the design margin tends to increase (the probability of premature failure decrease) because timeindependent failure supplants time-dependent failure by creep rupture. This analysis focused on the creep range and so only considered creep rupture. Therefore, as the temperature decreases the failure probabilities considered here will tend to decrease towards zero as creep rupture becomes essentially impossible. If the lower temperature range is of interest for a particular design then this analysis could be repeated including time-independent failure statistics basic on the observed variation in the material yield and tensile strengths.

- The margin decreases significantly for $316 \mathrm{H}$ above $800^{\circ} \mathrm{C}$. This may be related to the bias in the rupture database to more reasonable component temperatures, as the material has very little rupture life above $800^{\circ} \mathrm{C}$.

- For both materials there is some variation in the computed reliability even away from the extreme low and high ends of the temperature range considered in this analysis. These variations may be related to the difference between the ASME allowable stress $S_{m t}$, which considers rupture, tertiary creep, and strain accumula- 
- Experimental rupture data

- GP Fitting mean

- - GP 95\% prediction band

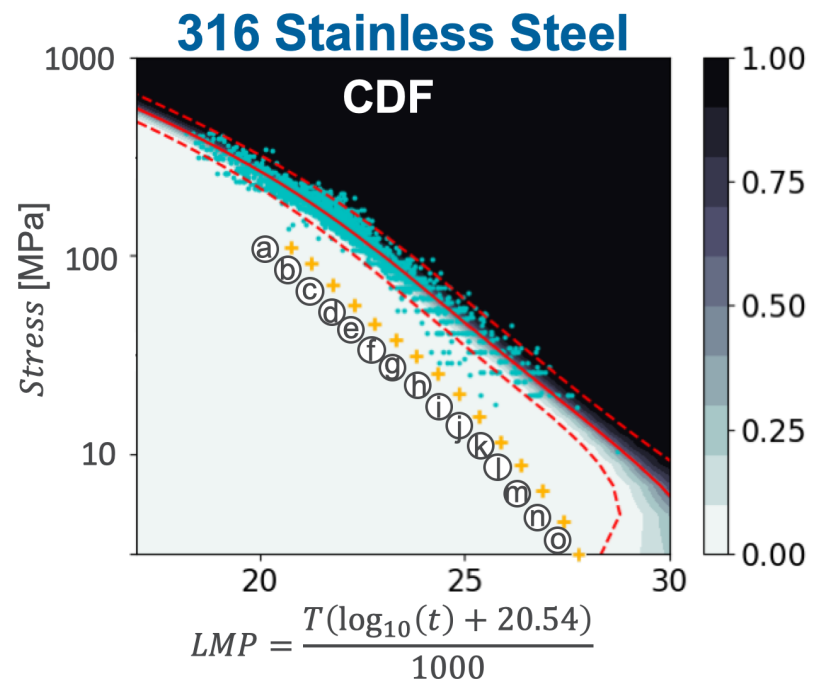

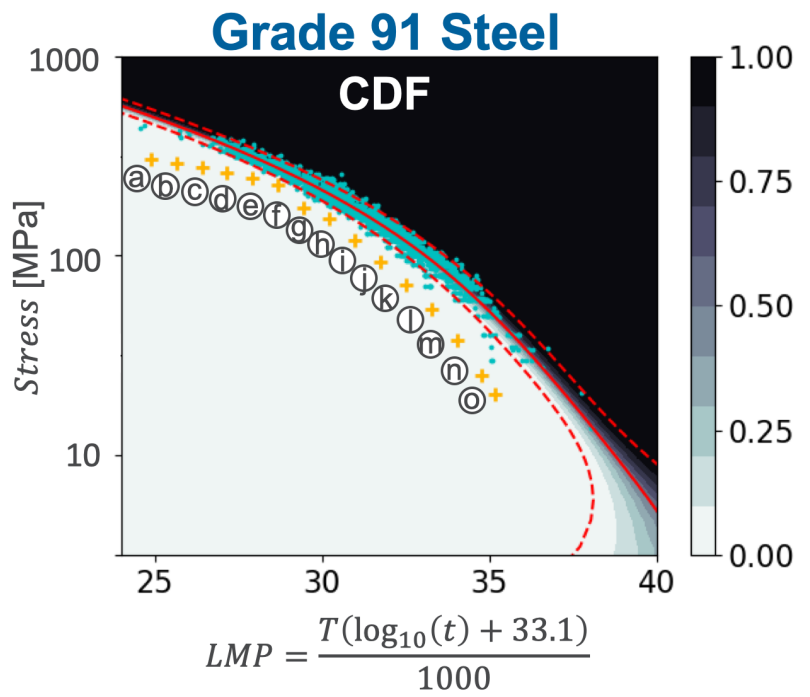

Figure 2.9: Rupture data along with a yellow scatterplot that shows the mean stress and mean LMP at each evaluated temperature in Table 2.2 and Table 2.3.

tion metrics, versus the rupture-only statistical analysis used here. The very small probabilities under consideration here may also contribute to these variations it would require a far larger rupture database to sample probabilities below $10^{-4}$ than exists currently, even for these two well studied materials. 
Comprehensive Margin Assessment of the ASME Section III, Division 5, Class A Primary Load Design Rules

September 2021

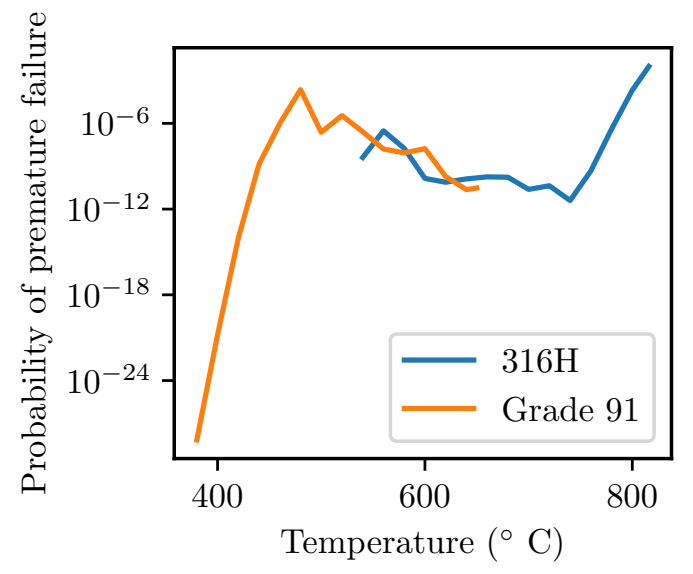

Figure 2.10: Probabilities from Tables 2.2 and 2.3 plotted as a function of temperature. 
Comprehensive Margin Assessment of the ASME Section III, Division 5, Class A Primary Load Design

Rules

September 2021

\section{Improved Gaussian Process Modeling of Creep Rupture Data}

\subsection{Overview}

The previous chapter uses Gaussian process regression to provide a statistical model linking the $(\log )$ stress to the Larson-Miller parameter, which in turn encapsulates the effects of temperature and time. The Larson-Miller parameter is

$$
L M P=T\left(\log _{10} t_{R}+C\right)
$$

with $T$ the absolute temperature, $t_{R}$ the rupture time, and $C$ a material-dependent parameter.

For the margin analysis of the Division 5 primary load rules we fixed the value of $C$ to the value that provides the best log-linear correlation between the Larson-Miller parameter and the $\log$ stress, both calculated from the experimental rupture database. This procedure has two drawbacks:

1. It means that our new approach based on non-parametric Gaussian process regression still partly relies on the traditional polynomial regression. The value of $C$ will depend on the order of the polynomial regression, introducing an unwanted additional hyperparameter.

2. This approach does not account for batch-to-batch variation in the value of the LarsonMiller $C$ parameter. In actuality this parameter can vary not only between materials but also between different heats and product forms for a single material.

This chapter derives a statistical calibration process based on Gaussian process regression that allows the Larson-Miller parameter $C$ to be a random variable. This new regression model overcomes both of the deficiencies of the previous approach.

\subsection{Gaussian Process Regression}

A covariance function $C\left(\boldsymbol{x}_{i}, \boldsymbol{x}_{j}\right)=\operatorname{cov}\left[f\left(\boldsymbol{x}_{i}\right), f\left(\boldsymbol{x}_{j}\right)\right]$ fully-defines the standard zero-mean Gaussian process [15]. This covariance function defines the stochastic function $f(\boldsymbol{x})$ over the data $\boldsymbol{x}$ by defining the covariance between two data points $\boldsymbol{x}_{i}$ and $\boldsymbol{x}_{j}$. The covariance function (for the zero-mean process) can be viewed as the kernel of a convolution integral and so is often called the kernel of the Gaussian process.

The covariance function will include hyperparameters that must be fit to the data. A common regression strategy is to find the maximum likelihood estimate of these parameters - the values of the parameters that maximize the likelihood of the model predictions versus the observed data. Statistical inference methods can also include white noise superimposed on the base model representing random measurement errors or other uncertainties. This makes the regression problem to find the parameter values that maximizes the marginal likelihood of

$$
y=f(\boldsymbol{x})+\epsilon
$$

where $\epsilon$ represents white noise with mean of zero and variance of $\sigma_{n}^{2}$. The log likelihood of a given dataset is $[15]$

$$
\ln p(y \mid \boldsymbol{x}, \theta)=-\frac{1}{2} y_{i} C_{i j}^{-1} y_{j}-\frac{1}{2} \ln \left|C_{i j}\right|-\frac{N}{2} \ln (2 \pi)
$$




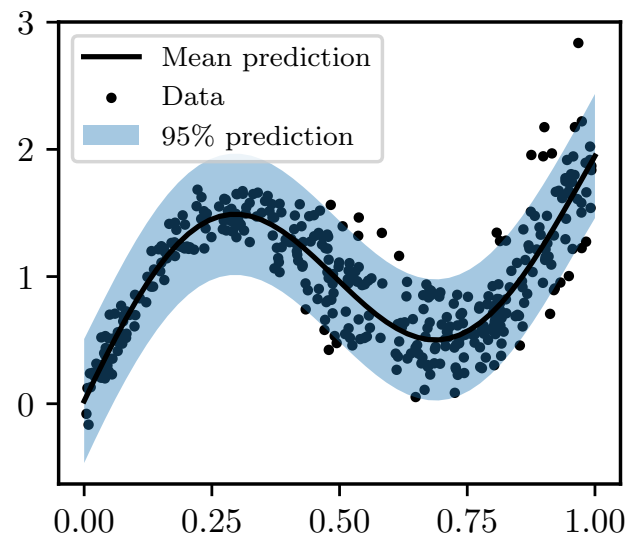

Figure 3.1: Example of Gaussian process regression.

where $\theta$ is the set of hyperparameters, $C_{i j}$ is the matrix containing the values of the covariance function evaluated at each pair of data points $x_{i}$ and $x_{j}$ and $N$ is the number of data points. Maximizing this function with respect to the kernel parameters provides the best (most likely) model.

As an example, consider the common case of a radial basis function kernel for scalar data, including the white noise directly in the definition of the kernel

$$
C\left(x_{i}, x_{j}\right)=\sigma_{f}^{2} \exp \left(-\frac{\left(x_{i}-x_{j}\right)^{2}}{2 l^{2}}\right)+\sigma_{n}^{2} \delta_{i j}
$$

This kernel has three hyperparameters - the process variance $\sigma_{f}^{2}$, the noise variance $\sigma_{n}^{2}$, and the process length scale $l$. Figure reffig:ex-gp illustrates Gaussian process regression by finding the parameters that maximize the likelihood of the data, generated by the model

$$
y=A \sin (2 \pi f x)+2 B x^{c}
$$

where $A, f, B$, and $c$ are all normal random variables with mean of 1.0 and scale 0.075 . In generating the data we further add white noise to the observations with scale 0.01 . The best fit Gaussian process regression line accurately captures the average response of the data.

In addition to providing non-parametric regression, statistical estimates of the uncertainty in the regression are an inherent part of Gaussian process models. Given a division of input data into the training and prediction sets, i.e.

$$
x=\left[\begin{array}{c}
x_{\text {training }} \\
x_{\text {prediction }}
\end{array}\right]
$$

the predicted outputs $y_{\text {prediction }}$ have a multivariate normal distribution with expected value

$$
\mathrm{E}\left[y_{\text {prediction }}\right]=C_{\text {prediction,training }} \cdot C_{\text {training,training }}^{-1} \cdot y_{\text {training }}
$$

and covariance

$$
\operatorname{cov}\left[y_{\text {prediction }}\right]=C_{\text {prediction,prediction }}+C_{\text {prediction,training }} \cdot C_{\text {training,training }}^{-1} \cdot C_{\text {training,prediction }} .
$$


So the model provides both the average (best) deterministic regression as well as the statistics needed to construct prediction bounds. Figure 3.1 further illustrates a $95 \%$ prediction interval on the regression.

\subsection{Gaussian Process Model for Creep Rupture}

Given the advantages of Gaussian process regression we apply it in Chapter 2 to predicting and extrapolating creep rupture test data using the Larson-Miller parameter to combine the effects of temperature and time. The regression problem can be posed as finding a regression model that correlates the log stress to the Larson-Miller parameter:

$$
\log _{10} \text { stress }=f(L M P)+\varepsilon
$$

where we again include white noise on the observations.

The challenge in directly applying the GP regression as described above is that the Larson-Miller parameter depends on $C$ which we now wish to represent as an additional random variable. Fortunately, there is a analytic solution, described as a kernel function, for the statistics of Gaussian process with a radial basis (squared exponential) kernel with uncertain inputs, provided the inputs are normally distributed [16, 17]. Applying this solution to Eq. 3.9 yields

$$
C\left(x_{i}, x_{j}\right)=\sigma_{f}^{2} \frac{\exp \left[-\frac{1}{2}\left(T_{i}\left(\mu_{C}+\log _{10} t_{R, i}\right)-T_{j}\left(\mu_{C}+\log _{10} t_{R, j}\right)\right)^{2} /\left(l+\left(T_{i}^{2}+T_{j}^{2}\right) \sigma_{C}^{2}\right)\right]}{\sqrt{1+\left(T_{i}^{2}+T_{j}^{2}\right) \sigma_{C}^{2} / l}}+\delta_{i j} \sigma_{n}^{2}
$$

where $\mu_{C}$ is the mean of the Larson-Miller $C$ parameter, $\sigma_{C}^{2}$ is the variance of the LarsonMiller $C$ parameter, and the input data $x_{i}$ is a tuple of the experimental temperature $T_{i}$ and rupture time $t_{R, i}$. This new kernel has five hyperparameters $-l, \sigma_{f}, \mu_{C}, \sigma_{C}^{2}$, and $\sigma_{n}$ - to infer using the process outlined in the previous section. The new GP model accounts for batch variation in the $C$ parameter described by a normal distribution with mean $\mu_{C}$ and variance $\sigma_{C}$, experimental noise with $\sigma_{n}$, and the non-parametric regression of the Larson-Miller parameter against the rupture stress with $l$ and $\sigma_{f}$. This new regression model improves on the fixed- $C$ version used in Chapter 2 and has advantages over classical Larson-Miller polynomial regression as well.

\subsection{Examples}

This section illustrates the advantages of the new, variable- $C$ GP regression technique to interpolate and extrapolate creep rupture data by comparing the new approach to traditional Larson-Miller polynomial regression using creep rupture databases for several high temperature materials.

To compare the approaches we fit both a GP and a classical regression model using the same data. Sections 3.2 and 3.3 describe the approach used to fit the GP model. The classical model is polynomial regression over the database, using the value of $C$ that provides the best regression model as measured by the coefficient of determination. The classical process requires selecting the polynomial order for the regression. To avoid overfitting, for each 


\begin{tabular}{c|cccc} 
& \multicolumn{2}{|c}{ Polynomial } & \multicolumn{2}{c}{ Gaussian process } \\
& MRE & Inside 95\% & MRE & Inside 95\% \\
\hline $316 \mathrm{H}$ & 0.09920 & $95.07 \%$ & 0.09531 & $95.18 \%$ \\
Grade 91 & 0.06143 & $94.91 \%$ & 0.06116 & $94.87 \%$ \\
A617 & 0.1701 & $95.68 \%$ & 0.1601 & $96.25 \%$ \\
A740H & 0.0464 & $94.33 \%$ & 0.0358 & $92.67 \%$
\end{tabular}

Table 3.1: Summary of the 10-fold cross-validation test, comparing the GP and polynomial regression approaches.

material we use either a linear model if the Pearson coefficient $R$ for the linear regression satisfies $R^{2}>=0.97$. Otherwise we use a quadratic model.

To test the robustness of the two approaches we assess the models using a 10-fold cross validation. We repeat the regression process 10 times for each model, for each material. Each time we reserve $1 / 10$ of the rupture data and calibrate the model against the remaining 9/10. Then, the process records the accuracy of the regression by calculating the mean relative error against the remaining 1/10 of the dataset as well as the percentage of the validation data that falls within a $95 \%$ prediction bound based on the calibration dataset. We repeat this process 10 times, so that each chunk of the rupture data serves as the validation set once. Finally, the process reports the averaged error metrics over the 10 separate trials.

Table 3.1 reports the results of this comparison for four materials: $316 \mathrm{H}$ stainless steel, Grade 91 steel, the Ni-based alloy 617, and the Ni-based alloy 740H. The first two materials are well-studied materials with a significant rupture database. Alloy 617 is another wellstudied alloy, but with a comparatively smaller rupture database than $316 \mathrm{H}$ and Grade 91 . Finally, Alloy $740 \mathrm{H}$ is a newer material with a smaller rupture database. Table 3.1 provides the results of the comparison.

Figures 3.2-3.5 visually compare the regression approaches. Each figure plots the rupture data using the best-fit, mean value of $C$ to calculate the Larson-Miller parameter, the mean prediction, and a $95 \%$ prediction interval for each regression model, for each material. Each figure shows the classical and GP approaches side-by-side, instead of on a single plot, because the two approaches can result in different best, mean values of $C$, which in turn shift the plotted rupture data.

Overall, the Gaussian process regression produces a more accurate model with lower relative error than the traditional Larson-Miller correlation. The reason can be seen in Figures $3.2,3.4$, and 3.5. A low order polynomial does not accurately describe the general trends in the Larson-Miller data for these materials and so the non-parametric GP regression better correlates the data. The improved correlation does not substantially diminish the ability of the model to predict future test data, i.e. the percentage of validation data points inside the 95\% prediction interval is about the same for both the polynomial and GP methods.

\subsection{Summary}

This chapter demonstrates the benefits of the new Gaussian process method over both traditional Larson-Miller polynomial regression and the fixed- $C$ Gaussian process used in the 


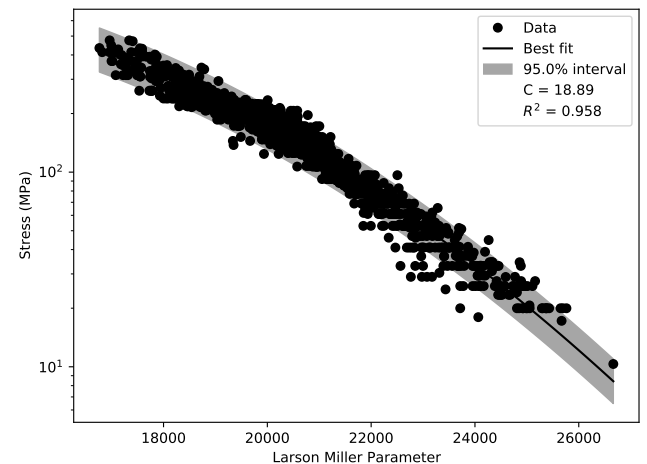

(a) Polynomial regression

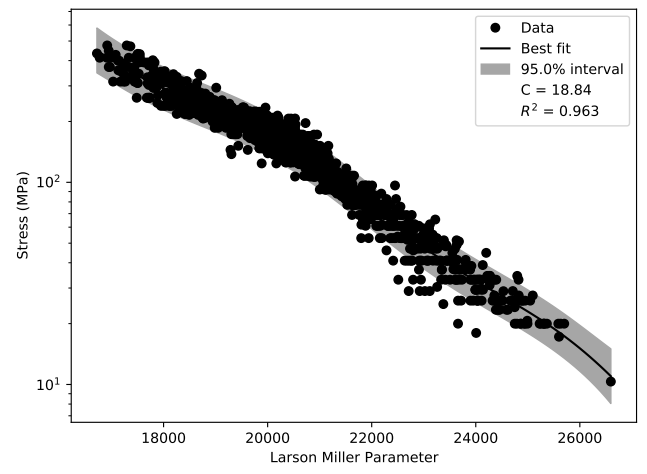

(b) Gaussian process regression

Figure 3.2: Comparison between classical Larson-Miller polynomial regression and the Gaussian process regression model for $316 \mathrm{H}$.

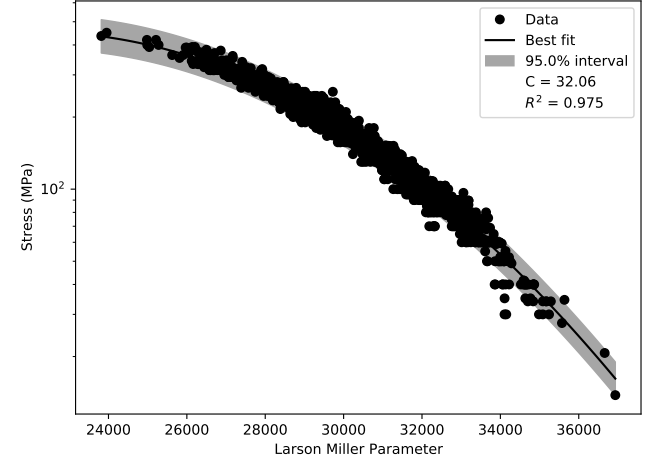

(a) Polynomial regression

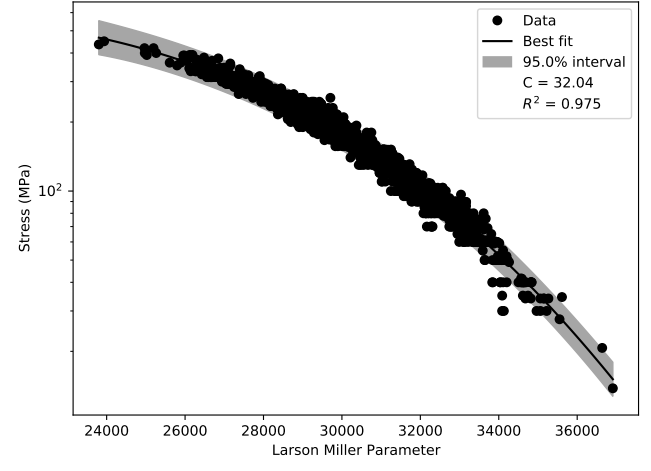

(b) Gaussian process regression

Figure 3.3: Comparison between classical Larson-Miller polynomial regression and the Gaussian process regression model for Grade 91.

previous chapter. We recommend the variable- $C$ process be used in future statistical analyses of creep rupture. The GP correlation could also compete with traditional Larson-Miller regression, including the more sophisticated region-split [18] and batch- $C$ variants actually used by ASME to set design creep rupture correlations. The primary advantage of the GP approach is that it accounts for all the effects captured by the current ASME method without requiring the user to select the order of polynomial regression, the number and location of region splits, and the heat of each material sample. Moreover, the GP regression is nonpolynomial and so the more flexible, non-parametric form may simply fit the data better than a polynomial correlation. 


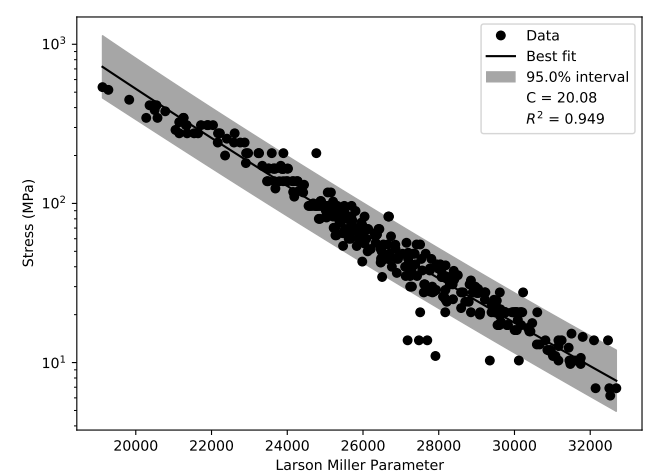

(a) Polynomial regression

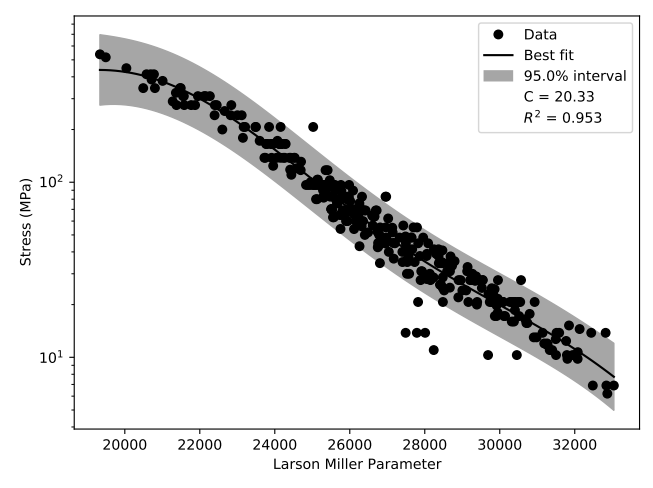

(b) Gaussian process regression

Figure 3.4: Comparison between classical Larson-Miller polynomial regression and the Gaussian process regression model for Alloy 617.

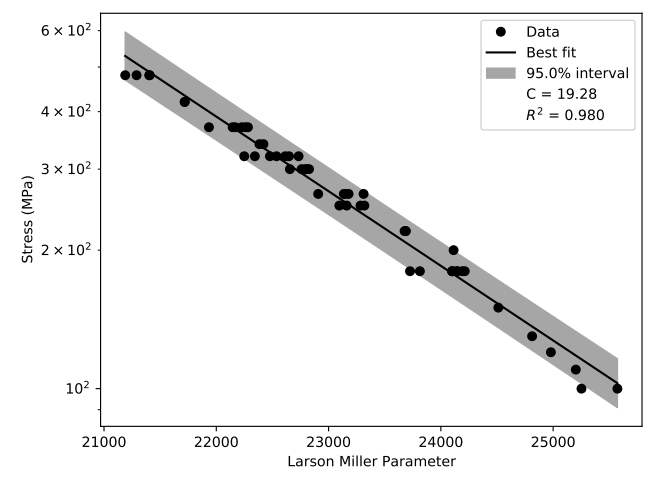

(a) Polynomial regression

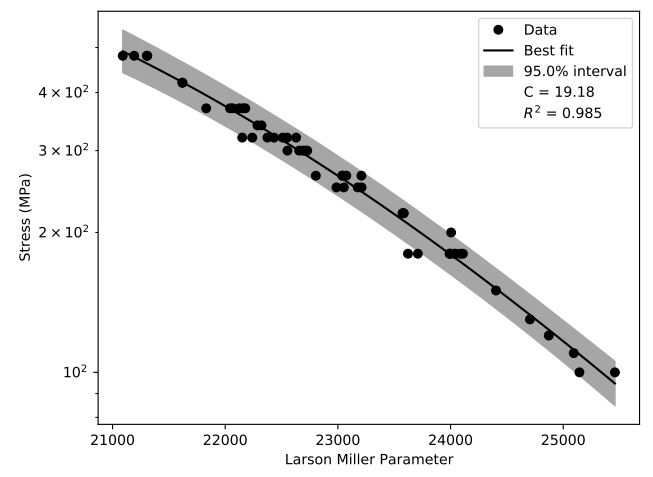

(b) Gaussian process regression

Figure 3.5: Comparison between classical Larson-Miller polynomial regression and the Gaussian process regression model for Alloy $740 \mathrm{H}$. 


\section{Conclusions}

\subsection{Meaningful Probabilities}

The probabilities assessed here are quite small - significantly less than $10^{-4}$ for most temperatures. It is worth considering if values this small are meaningful relative to the size of the underlying experimental databases.

One advantage of the probabilistic assessment method described here is that it is a consistent tool for combining small probabilities. Consider calculating the probability of failure as a product of independent events describing the component loading, the minimum creep rate, and the rupture time:

$$
p_{f}=p\left(t=t^{\prime}\right)=\int_{L} \int_{\sigma} p\left(t^{\prime} \mid \sigma\right) p(\sigma \mid L) p(L) d \sigma d L
$$

This equation connects the loading statistics $L$, the stress state $\sigma$, controlled by the minimum creep rate, and the failure time $t$, assuming that the three distributions are independent. Even if the individual probabilities are very small, the total probability describing the component service life is still meaningful, again given the assumption that the distributions are independent.

However, consider a simple case where the loading is well-characterized, there are no significant thermal stresses, and the structure does not have a redundant load path, meaning the creep deformation statistics do not significantly affect the component stress distribution. In this case, the probability of premature failure is controlled by the creep rupture statistics. Table 4.1 lists the size of the creep rupture database for the four materials considered in this report. Even the largest databases only have a few thousand tests. This means that for probabilities less than $10^{-4}$ it is unlikely that a single experiment actually samples the relevant tail of the rupture distribution. For this case then, there may be no significant difference between probabilities smaller than $10^{-4}$ as they all express a previously unobserved occurrence.

\subsection{Summary}

This report describes a comprehensive margin assessment procedure applied to assess the design margin of the Section III, Division 5, Subsection HB, Subpart B primary load design rules for Class A components. The assessment calculates the probability that the component fails via creep rupture before the ASME design life using the steady-state stress distribution. This process mirrors the ASME primary load assessment procedure. However, the approach

\begin{tabular}{cc}
\hline Material & Number of rupture tests \\
\hline $316 \mathrm{H}$ & 1764 \\
Grade 91 & 2047 \\
A617 & 348 \\
A740H & 54 \\
\hline
\end{tabular}

Table 4.1: The size of the rupture database for the four materials considered in this report. 
described here is fully statistical, accounting for variations in the component loading, creep deformation, and creep failure properties. We apply the margin assessment approach to provide a comprehensive, temperature-dependent margin assessment for the ASME primary load rules for two materials: 316H stainless steel and Grade 91 steel. The key conclusions of the margin assessment study are:

1. The Code design rules produce components with a substantial design margin against creep rupture. With one exception $(316 \mathrm{H}$ at the maximum ASME allowable temperature) the probability of premature rupture remains substantially less than $10^{-4}$ over the full design life. While further investigation into 316H Code rupture data is worthwhile, this exception is not significant for design as in practice $816^{\circ} \mathrm{C}$ is too hot to viably operate a $316 \mathrm{H}$ component.

2. Given the size of the creep rupture testing databases available for the Code materials probabilities below $10^{-4}$ likely cannot be reasonably distinguished as the entire experimental database would not contain a single specimen sampling the event of interest. As such, any chance of premature failure for properly-design ASME components would exist in the ill-characterized tail of the failure distribution representing as-yet unobserved events.

3. For the temperature range most relevant for future reactor designs, away from very high and very low temperature use cases, the ASME design margin is roughly consistent between the two materials.

4. The design margin against rupture increases rapidly at lower temperatures. However, this simply reflects the decreased likelihood of creep failure in the temperature regime where time-independent failure modes will begin to become significant.

5. At least for $316 \mathrm{H}$, the margin against rupture decreases rapidly close to the material's maximum use temperature. This could be attributed to gaps in the underlying creep rupture database. The $316 \mathrm{H}$ allowable stresses will be under discussion at ASME with a view to extending the maximum design life to 500,000 hours and so this issue can be discussed during that process.

6. Within the design range, the margin against rupture is not constant as a function of temperature. The full ASME primary load allowable stresses include tertiary creep and creep deformation criteria and so this variation may be caused by regions in time/temperature space controlled by properties other than rupture. However, the design method could be optimized to provide a more uniform margin.

With the possible exception of the $316 \mathrm{H}$ allowable stresses above $800^{\circ} \mathrm{C}$, all these issues can be characterized as design Code optimizations and not deficiencies. The $316 \mathrm{H}$ allowable stresses in this temperature range are a low priority as the maximum use temperature for the material is $816^{\circ} \mathrm{C}$, mean the reduced margin region only covers $16^{\circ} \mathrm{C}$ in a regime where the material has very low creep rupture strength.

The probabilistic assessment approach developed here is complete, but a few incremental improvements are possible: 
1. The component analysis should be repeated with the improved Gaussian process model allowing for heat-to-heat variations in the Larson-Miller $C$ parameter. While this should be done for consistency, in practice there would be very little change in the final calculated probabilities of failure as the fixed- $C$ and stochastic- $C$ rupture correlations are still very similar.

2. The statistical minimum creep rate model could be improved. The current approach attempts to correlate isothermal estimates of the statistics describing the prefactor and stress exponent of a power law creep model, taken from log-linear regression models against the minimum creep rate data. A better approach would be to reuse the Gaussian process model for rupture developed in Chapter 3 by correlating the stress to the temperature and $1 / \dot{\varepsilon}_{\min }$, which has units of time. The only change required to the Stokes flow model would be to adopt a compatible nonlinear viscosity model. This approach might better correlate the data, but would also provide a cleaner temperaturedependent statistical model.

3. The current work deliberately only considers creep rupture failure. As described in Chapter 1, creep rupture is the only actual failure model among the ASME allowable stress criteria. However, a fully-consistent margin assessment would consider all three time dependent design criteria used in construction the ASME allowable stress: rupture, time-to-tertiary creep, and time to $1 \%$ strain. The current method could easily be extended to cover these additional criteria by developing statistical models for the time-to-tertiary and time to $1 \%$ strain data.

There is also one more significant defect in our current approach - it assumes that each of the loading, deformation, and failure probability distributions describe independent events. Decoupling the loading from the material creep rate and creep rupture time is reasonable, as there is no a prior reason why the component loading and material properties should be correlated. However, there is almost certainly a correlation between the minimum creep rate and the material creep strength. The challenges in qualifying this correlation are that (1) often abstracted data does not consistently identify the material heat for both the creep rate and creep life measurements and (2) there are significantly fewer minimum creep rate measurements than rupture time measurements, as creep rate data requires an instrumented creep test. However, an effort aimed at reconstructing the data required to examine the correlation between creep rate and strength would be worthwhile, as it could improve statistical service life predictions of the type discussed here.

Either the current or improved approaches could be applied to assess the margin for the other Class A ASME materials or for a wider variety of components. There are no obstacles to completing these assessments, except potentially in reconstructing the rupture and deformation database for $2.25 \mathrm{Cr}-1 \mathrm{Mo}$ and $304 \mathrm{H}$. For larger components requiring 3D analysis the Monte Carlo structural analysis would be computationally expensive but it would be reasonably achievable even on moderately sized cluster computers.

Finally, the statistical life assessment method developed here could form the basis of a statistical design approach. The current assessment method could be used for probabilistic design. However, the Monte Carlo structural analysis is likely too computationally expensive 
and time-consuming to use in engineering practice except for either very simple geometries or for a limited number of critically important components.

A more practical engineering approach would be to replace the statistical structural analysis with a factored deterministic analysis, in the spirit of a Load and Resistance Factor Design method. One challenge in developing such an approach is that for high temperature design there are three critical probability distributions - applied loads, material deformation, and material resistance - versus the two distributions - applied load and material resistance - embedded in current LRFD approach like the method described in the AISC Steel Construction Manual. A second challenge would be developing the required load, deformation, and resistance factors and accounting for temperature dependence; temperature-dependent is another challenge not addressed by current LRFD methods. A large database of realistic, representative statistical creep analyses would be invaluable in constructing the design factors, meaning analyses used for a broader margin assessment of the current Code could be reused in developing future probabilistic creep design methods.

This report does not address the question of determining what an adequate design margin for ASME Class A components should be. We advocate for a particular measurement of the design margin, the probability of premature failure, demonstrate that the current Code rules produce components with premature failure probabilities generally much less than $10^{-4}$, and suggest that probabilities less than $10^{-4}$ may not have significant meaning, given the size of the available creep rupture test databases. However, determining an acceptable design margin is beyond the scope of this report as it is a broader social question that should involve the cognizant ASME Code Committees, regulators, and future plant owner/operators. 
Comprehensive Margin Assessment of the ASME Section III, Division 5, Class A Primary Load Design Rules

September 2021

\section{Acknowledgements}

The research was sponsored by the U.S. Department of Energy (DOE), under Contract No. DE-AC02-06CH11357 with Argonne National Laboratory, managed and operated by UChicago Argonne LLC, and Contract No. DE-AC07-05ID14517 with Idaho National Laboratory (INL), managed and operated by Battelle Energy Alliance LLC. Programmatic direction was provided by the Advanced Reactor Technologies (ART) Program of the Office of Nuclear Reactor Deployment of the Office of Nuclear Energy (NE). The authors gratefully acknowledge the support provided by Sue Lesica of DOE-NE, Federal Lead, ART Advanced Materials, Diana Li of DOE-NE, Federal Manager, ART Gas-Cooled Reactors (GCR) Campaign, and Gerhard Strydom of INL, National Technical Director, ART GCR Campaign. 

Comprehensive Margin Assessment of the ASME Section III, Division 5, Class A Primary Load Design

Rules

September 2021

\section{Bibliography}

[1] Welding Research Council. Recommended practices in elevated temperature design: a compendium of breeder reactor experiences (1970-1987): Volume 1 - current status and future directions. Technical report, WRC, 1991.

[2] P. J. Holt and R. A.W. Bradford. Application of probabilistic modelling to the lifetime management of nuclear boilers in the creep regime: Part 1. International Journal of Pressure Vessels and Piping, 95:48-55, 2012. ISSN 03080161. doi: 10.1016/j.ijpvp.2012. 05.007. URL http://dx.doi.org/10.1016/j.ijpvp.2012.05.007.

[3] R. A.W. Bradford and P. J. Holt. Application of probabilistic modelling to the lifetime management of nuclear boilers in the creep regime: Part 2. International Journal of Pressure Vessels and Piping, 111-112:232-245, 2013. ISSN 03080161. doi: 10.1016/j. ijpvp.2013.08.002. URL http://dx.doi.org/10.1016/j.ijpvp.2013.08.002.

[4] N. A. Zentuti, J. D. Booker, R. A.W. Bradford, and C. E. Truman. A review of probabilistic techniques: towards developing a probabilistic lifetime methodology in the creep regime. Materials at High Temperatures, 34(5-6):333-341, 2017. ISSN 09603409. doi: 10.1080/09603409.2017.1371933.

[5] James Hensman, N Fusi, and Neil D. Lawrence. Gaussian Processes for Big Data. In Proceedings of the Twenty-Ninth Conference on Uncertainty in Artificial Intelligence, pages 282-290, 2013. ISBN 978-1-4503-1285-1. doi: 10.1162/089976699300016331. URL http://auai.org/uai2013/prints/papers/244.pdf.

[6] Christopher KI Williams and Carl Edward Rasmussen. Gaussian processes for machine learning, volume 2. MIT press Cambridge, MA, 2006.

[7] ASME. BPVC Section III-Rules for Construction of Nuclear Facility Components Division 5. ASME BPVC.III.5-2017, 2017.

[8] Arif Masud and Jae Hyuk Kwack. A stabilized mixed finite element method for the incompressible shear-rate dependent non-Newtonian fluids: Variational Multiscale framework and consistent linearization. Computer Methods in Applied Mechanics and Engineering, 200(5-8):577-596, 2011. ISSN 00457825. doi: 10.1016/j.cma.2010.08.012.

[9] Andrea Nicolas, Mark C Messner, and T L. Sham. Initial development of a high temperature life prediction method directly accounting for variability in material properties (ANL-ART-203). Technical report, Argonne National Laboratory, Illinois, United States, 2020. URL https://www.osti.gov/biblio/1658590.

[10] John W. Peterson, Alexander D. Lindsay, and Fande Kong. Overview of the incompressible Navier-Stokes simulation capabilities in the MOOSE framework. Advances in Engineering Software, 119:68-92, 2018. ISSN 18735339. doi: 10.1016/j.advengsoft.2018. 02.004 .

[11] Cody J. Permann, Derek R. Gaston, David Andrš, Robert W. Carlsen, Fande Kong, Alexander D. Lindsay, Jason M. Miller, John W. Peterson, Andrew E. Slaughter, Roy H. 
Stogner, and Richard C. Martineau. MOOSE: Enabling massively parallel multiphysics simulation. SoftwareX, 2020. ISSN 23527110. doi: 10.1016/j.softx.2020.100430.

[12] Thomas J.R. Hughes, Leopoldo P. Franca, and Marc Balestra. A new finite element formulation for computational fluid dynamics: V. Circumventing the babuška-brezzi condition: a stable Petrov-Galerkin formulation of the stokes problem accommodating equal-order interpolations. Computer Methods in Applied Mechanics and Engineering, 59(1):85-99, 1986. ISSN 00457825. doi: 10.1016/0045-7825(86)90025-3.

[13] Benoit Revil-Baudard and Oana Cazacu. New three-dimensional strain-rate potentials for isotropic porous metals: Role of the plastic flow of the matrix. International Journal of Plasticity, 2014. ISSN 07496419. doi: 10.1016/j.ijplas.2014.04.003.

[14] David M Levine, David F Stephan, Timothy C Krehbiel, and Mark L Berenson. Statistics for Managers using Microsoft Excel. Prentice Hall, New Jersey, 5th edition, 2008. ISBN 0-536-04080X.

[15] Carl Edward Ramussen and Christopher K. I. Williams. Gaussian Processes for Machine Learning, volume 7. MIT Press, Boston, 2006. ISBN 026218253X.

[16] Agathe Girard. Approximate methods for propagation of uncertainty with Gaussian process models. Doctoral dissertation, University of Glasgow, 2004. URL http://citeseerx. ist.psu.edu/viewdoc/download?doi=10.1.1.66.1826\{\&\}rep= rep1\{\&\}type=pdf.

[17] Patrick Dallaire, Camille Besse, and Brahim Chaib-draa. Learning Gaussian Process Models from Uncertain Data. In Chi Sing Leung, Minho Lee, and Jonathan H. Chan, editors, Neural Information Processing, pages 433-440, Bangkok, Thailand, 2009. Springer. ISBN 9783642106767.

[18] Kazuhiro Kimura and Yukio Takahashi. Evaluation of Long-Term Creep Strength of ASME Grades 91, 92, and 122 Type Steels. In ASME 2012 Pressure Vessels and Piping Conference, pages 309-316, 2012. 



\section{Argonne}

\section{Applied Materials Division}

Argonne National Laboratory

9700 South Cass Avenue, Bldg. 212

Argonne, IL 60439

www.anl.gov 